\section{EMBRYARIDDLE \\ Aeronautical University}

SCHOLARLY COMMONS
International Journal of Aviation, Aeronautics, and Aerospace

\title{
Satellite Maintenance: An Opportunity to Minimize the Kessler Effect
}

Bettina M. Mrusek Dr.

Embry Riddle Aeronautical University, mrusekb@erau.edu

Follow this and additional works at: https://commons.erau.edu/ijaaa

Part of the Aviation and Space Education Commons, Maintenance Technology Commons, Other Astrophysics and Astronomy Commons, Propulsion and Power Commons, Space Vehicles Commons, and the Sustainability Commons

\section{Scholarly Commons Citation}

Mrusek, B. M. (2019). Satellite Maintenance: An Opportunity to Minimize the Kessler Effect. International Journal of Aviation, Aeronautics, and Aerospace, 6(2). https://doi.org/10.15394/ijaaa.2019.1323

This Position Paper is brought to you for free and open access by the Journals at Scholarly Commons. It has been accepted for inclusion in International Journal of Aviation, Aeronautics, and Aerospace by an authorized administrator of Scholarly Commons. For more information, please contact commons@erau.edu. 
Orbital debris is a growing problem, notably in the Low Earth Orbit (LEO) (Adilov, Alexander, \& Cunningham, 2018; Drmola \& Hubik, 2018). Satellites in this orbit provide crucial information about our planet's weather, atmosphere, and allows for global communication. Additionally, advances in technology have made the satellite launching process much simpler (Russell, 2017). Today's satellites are smaller, more efficient, and can be launched fairly easily, as evidenced by the emergence of constellations. However, these advances should not diminish the harshness of the space environment itself. There are many elements which can compromise satellite operation in space (National Aeronautics and Space Administration [NASA], 2018a). As a result, some satellites become inoperable prior to their scheduled end of life sequence which creates orbital debris. The projected number of satellite launches over the next decade further complicates this problem (Anselmo \& Pardini, 2017; Drmola \& Hubik, 2018; Russell, 2017). This has resulted with an unsustainable and financial wasteland of inactive satellites orbiting the Earth. The Kessler Effect, which refers to the cascading impact of satellite collisions in orbit culminating in complete debris saturation (making it uneconomical to launch satellites into orbit) (Cour-Palais \& Kessler, 1978) also poses a problem. As the number of satellites placed in orbit grows, so too does the potential for collisions, further increasing the likelihood of additional space debris as well as wasted resources. While there have been some efforts to minimize this problem, none have proved significantly fruitful as of yet. One area which has yet to be fully explored is the potential for satellite maintenance in orbit. While traditionally this has not been considered a viable option, advances in propulsion, advanced navigation, and robotics may provide an opportunity to perform maintenance and/or repair satellites in LEO. Using a modified causal loop diagram, the researcher will demonstrate how satellite maintenance can minimize the Kessler Effect and can be used in conjunction with other orbital debris mitigation efforts.

\section{Research Question}

RQ. How can satellite maintenance minimize the Kessler Effect?

Drmola and Hubik (2018) identifieds inactive satellites as a contributor to orbital debris (as seen in the figure below and noted by the arrow). If this element can be removed (or shifted to the active satellite group), it may have a measurable impact on the rate of collisions. Using previous research on the removal of inactive satellites as support, a modified causal loop diagram will be constructed using system dynamics software. Flowcharts will also be used to illustrate the relationships between the variables. 


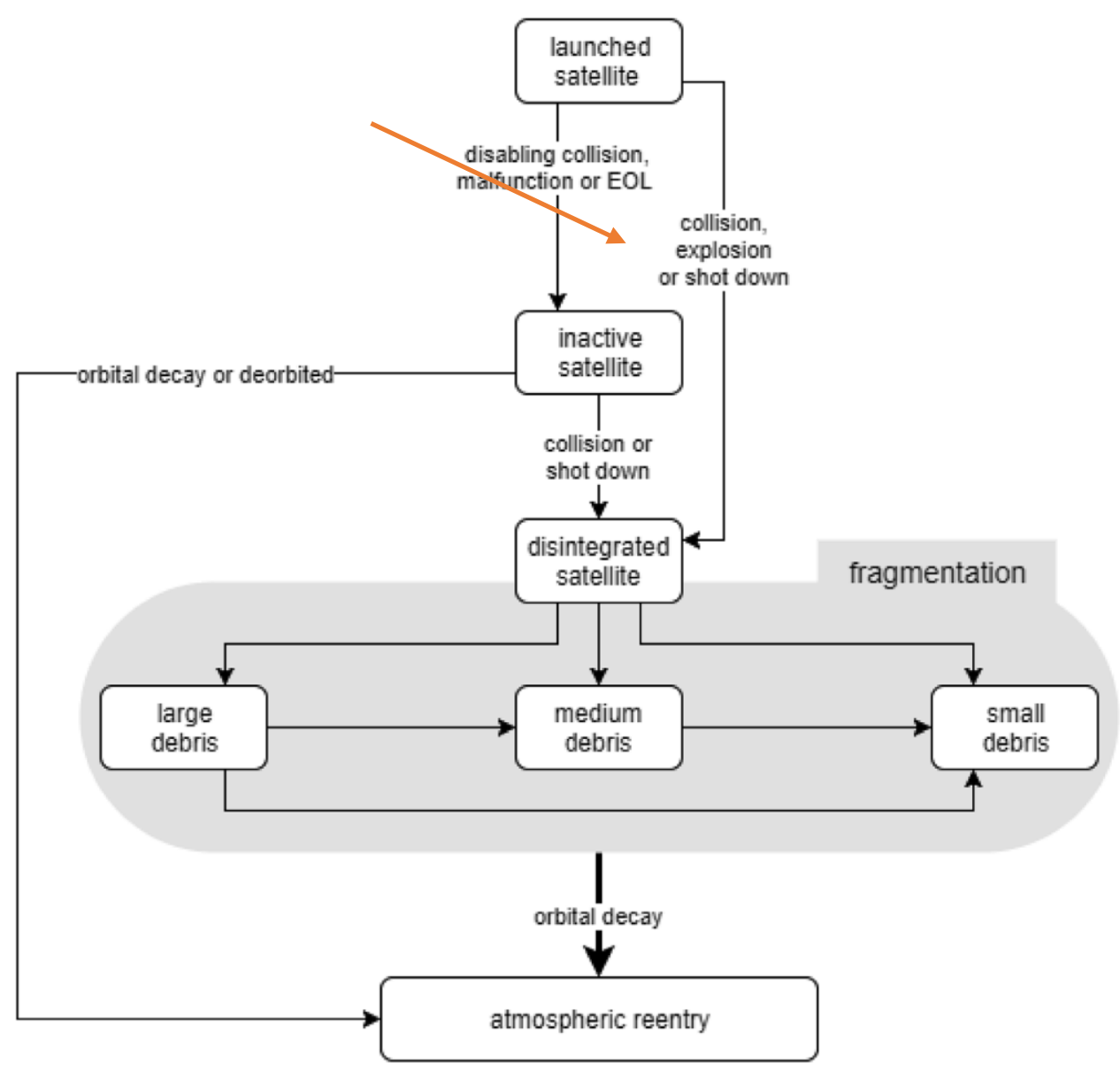

Figure 1. Flowchart of potential satellite and debris transitions. Adapted from "Kessler Syndrome: System Dynamics Model," by J. Drmola, and T. Hubik, 2018, Space Policy, 44/45, p. 32. doi:10.1016/j.spacepol.2018.03.003

\section{Literature Review}

To address the potential impact of satellite maintenance on the Kessler Effect, several areas were reviewed. First, the orbital debris environment and the resulting impact on the Kessler Effect. Next, current mitigation efforts were explored including satellite maintenance efforts, active debris removal, and the necessity of effective post-mission procedures. Finally, existing technological opportunities which support the potential for satellite maintenance and repair were explored. 


\section{Orbital Debris and the Kessler Affect}

The initial review focused on the orbital debris environment; data were analyzed related to the current number of man-made objects, which orbits they inhabit, and research related to the tracking and identification of these objects. In doing so, two key themes emerged; the validation of the orbital debris problem and the difficulties that exist in attempting to monitor the severity of the situation. This information provided the initial support, via quantifiable data and scholarly research, for the continued examination of the research question.

\section{Satellite Survivability}

According the European Space Agency (ESA), since 1957, there have been approximately 8,650 rocket launches that have carried satellites into space (2018b). Of that number, nearly 4700 are still in space, although only 1800 are still functioning. This means that out of all the satellites in space, only about $38 \%$ are operational. Given the nearly 60-year timeframe that has elapsed, it is reasonable that some of these satellites may have simply reached their scheduled end of life. Earth observation satellites, such as Landsat, can last up to 30 years (Kelly \& Holm, 2014) and CubeSats can last an estimated 25 years (Vavrin, Matney, \& Manis, 2017). However, many do not reach their scheduled end of life due to the challenges presented by the space environment. As a result, satellite survivability has become an area of interest. Some research efforts focus on the feasibility of extending current lifespans (Gonzalo, Domínguez, \& López, 2014), while others seek to optimize the cost structures while maintaining survivability (Khokhlachev, 2011). These research efforts demonstrate the need to promote long-term use of satellites in orbit. In doing so, the financial investment of satellite operations can be achieved and maintained, while space debris can be mitigated.

Another consideration is the potential growth of the satellite industry; for this growth will make long-term survivability a key element in the sustained use of the orbits surrounding our planet. In a recent report by Euroconsult, over 3,000 satellites are projected to be launched between 2017 and 2026 (Russell, 2017). This increase is notably due to advances in the small satellite industry, as well as the advent of constellations.

\section{Kessler Effect}

When discussing the growth of the satellite industry, and the potential impact on orbital debris, a key concept quickly rises to the surface; the Kessler Effect. The harshness and unpredictability of the space environment can have unintended consequences on satellites in orbit, such as collisions. When these objects collide, the debris that is subsequently created can have a cascading effect, causing other debris to then collide with it in orbit. This event was identified by prominent NASA scientist Donald Kessler, and as such, is now referred to as the 
Kessler Effect (Cour-Palais \& Kessler, 1978). The concern with such an event is that it will eventually reach a point where it is uneconomical to launch a satellite into the LEO due to the concentration of debris.

Some researchers have focused on prediction models, in an effort to quantify the severity of the problem (Drmola \& Hubik, 2018; Bonnal, Ruault, \& Desjean, 2013). In doing so, determinations or estimations on the number of satellites which need to be removed to stabilize the debris problem can be identified. Drmola and Hubik have theorized that the removal of eight inactive satellites per month, beginning in several decades, could stabilize the effect. Additionally, in a recent NASA report, future projections indicate that for every 100 satellites launched, 99 must be de-orbited when its mission is complete (NASA, $2018 \mathrm{~b}$ ). Both of these scenarios reduce the likelihood of future space debris, however, the challenge lies in the fact that debris which is currently in orbit continues to pose a threat.

\section{Mitigation Efforts}

With an understanding of the severity of the orbital debris problem, it is prudent to review current mitigation efforts. Both NASA and ESA, along with other international agencies, have identified methods for the mitigation of orbital debris. Given the mounting research on the effects of orbital debris, it is clear that significant efforts must be made on a global scale in order to ensure the long-term use of the orbits surrounding our planet. In 1997, Orbital Debris Mitigation Standard Practices, which were based on NASA-developed guidelines, were published by the U.S. government in an effort to create a standard set of practices (NASA, 2018b). However, in addition to general guidelines, other mitigationspecific efforts were needed such as satellite maintenance, active debris removal procedures, and effective post-mission procedures (ESA, 2018a).

\section{Satellite Maintenance}

Traditionally, maintaining or repairing satellites in orbit was simply unfeasible (Drmola \& Hubik, 2018). The costs associated with such a mission would far outweigh the benefits of the repair. However, advances in propulsion, advanced navigation, and robotics may provide an unprecedented opportunity. Given today's satellite environment, in terms of accumulating debris and potential growth, the ability to extend the lives of current or inactive satellites may be more profitable than previously thought. Both NASA and Defense Advanced Research Projects Agency (DARPA) are currently working on such missions.

NASA's project, Restore-L, seeks to provide in-orbit satellite servicing options (NASA, 2018c). Leveraging robotics, unmanned capabilities, and advanced sensors, Restore-L will have the capability to refuel existing satellites and perform fleet maintenance, which ultimately serve as debris mitigation efforts. The 
autonomous spacecraft is intended for use in LEO, although as its technology progresses, its capabilities may be extended to other orbits. The projected launch date for the spacecraft is 2020. This research demonstrates that satellite maintenance, in orbit, may be a real possibility in the foreseeable future.

DARPA is also working on a similar satellite servicing robotic system, although its intended use is for satellites in the geosynchronous orbit (DARPA, 2018). The program is currently in work and aims to demonstrate the potential for satellite servicing in orbits beyond LEO. Similar to NASA's Restore-L, DARPA's project would contribute to existing debris mitigation efforts by extending the current lifespans of satellites. The capabilities of the spacecraft include inspection, orbital correction, relocation, and upgrade installation (DARPA).

\section{Active Debris Removal}

In an effort to mitigate the problems associated with orbital debris, active debris removal (ADR) efforts have been an area of focus and research, similar to the study of the Kessler Effect. Some studies emphasized the importance and need for effective ADR efforts (Vavrin et al., 2017), while others have examined the progress and trends related to ADR (Bonnal et al., 2013). The latter study discovered that while ADR efforts are needed, they should be priority based. Debris which presents the highest probability to stabilize the Kessler Effect, for example, should be targeted. This, in turn, should drive ADR missions.

In addition to managing ADR efforts, specific removal methods have also been researched. Qi, Misra, and Zuo (2017) explored a double-tethered space tug system, while others examine the potential for the capture and disposal of debris (Forshaw et al., 2016). Government agencies are also exploring ADR methods. The ESA recently shifted its efforts to recapture a defunct Earth observation satellite, and instead is studying the potential for satellite servicing and active debris removal (Werner, 2018). The effectiveness of these missions is crucial to long-term debris mitigation procedures. ADR presents an opportunity to mitigate the growth of debris; when combined with other efforts, true stabilization of the Kessler Effect could be achieved.

\section{Post-Mission Procedures}

From a debris mitigation perspective, the end of mission procedures are just as important as the initial sequences. All missions must have a projected end point. When this time arrives, the system must be disposed of properly. When these sequences are not properly performed, debris results. This debris is unpredictable and creates the potential for collisions in space. Disposal scenarios will vary depending on the type and size of the satellite, but it is important to consider these decisions early in the development of the satellite to ensure proper hardware is installed (Hull, 2013). 
In an effort to preserve the space environment and ensure long-term use of the orbits surrounding our planet, many of the major satellite manufacturers today have established end of life disposal procedures. For example, OneWeb, a satellite manufacturer specializing in constellations, utilizes state of the art onboard GPS and propulsion systems to deorbit its satellites when it nears the intended service life (OneWeb, 2019). However, much of this can be attributed to the early work of NASA and other space agencies. In 1995, NASA was the first space agency to establish orbital debris mitigation guidelines, which included specific actions for the proper disposal of satellites (NASA, 2018a). This document became the cornerstone of orbital debris mitigation efforts and established procedures, and eventually paved the way for the Inter-Agency Space Debris Coordination Committee (IADC) in 2002, which currently includes 13 space agencies from countries around the world (IADC, 2016). Through a consolidated effort, these agencies have formed the framework for the proper identification and execution of debris mitigation procedures.

\section{Existing Technological Opportunities}

With the need for and established framework regarding the proper disposal of satellites examined, it is pertinent to identify the feasibility of such actions via existing technology applications. As noted above, the space environment is incredibly harsh. A key component in the successful implementation of debris mitigation efforts is the ability to identify and carry out specific procedures in orbit. There are many ways to accomplish these tasks: propulsion, advanced navigation and collision avoidance systems, robotics, and sensors. Each of these elements is needed to identify an inoperable satellite or man-made object, and then to perform the necessary adjustments.

Propulsion systems allow for maneuverability in the space environment. Advances in this technology have allowed for the emergence of smaller satellites, such as CubeSats, in the satellites market (Tummala \& Dutta, 2017). Considered micro-propulsion, these systems provide the appropriate thrust-to-power ratios needed to for orbit modifications and attitude control.

Another key component in debris mitigation is the ability to successfully identify and capture objects that are literally tumbling through space at incredible speeds. Accurate trajectory models must be employed to identify the object, and then to determine the directionality of its movement. Chu, Zhang, Zhang, Lu, and Sun proposed such a model using algorithms (2016). The model simulated a spacecraft which used collision avoidance in the ultra-close proximity of a failed satellite. This research demonstrates the potential for not only collision avoidance measures for operational satellites, but also the ability for maintenance spacecraft to determine the orbital dynamics of a tumbling satellite. With this information, the 
next phase of the debris mitigation procedure can be carried out: the capture of the tumbling satellite.

Given the recent emphasis on debris mitigation, and the potential for satellite maintenance, much research has been done on the use of robotic manipulators. This technology is essential for in-orbit satellite servicing. In a 2018 study by Valverde and Tsiotras, the use of dual quaternions (an advanced mathematical tool) was used to examine the relationship and complexities that exist between spacecraft control and fixed-based robotic manipulation. Studies such as these contribute to the growing body of knowledge related to the use of robotics in the service and maintenance of satellites in orbit.

\section{Research Analysis}

To perform the analysis and determine whether or not satellite maintenance could minimize the Kessler Effect, data obtained from the literature review, causal loop diagrams, and flowcharts were used. Comparisons were made between the previous work of Drmola and Hubik (2018) and the author's findings. Simantics software was used to create the new causal loop diagrams, which accounted for satellite maintenance. Simantics is a modelling and simulation software package that is useful in examining non-linear relationships (Simantics, 2019). Given the cascading effect of collisions resulting from orbital debris and the nonlinear relationship between satellites and debris, this software was an appropriate choice for the analysis of the research problem. While actual calculations were not used, the ability to create causal loop diagrams which illustrate the relationships between the variables was incredibly useful in examining the problem and supports the need for additional research in this area.

As seen in Figure 2, Drmola and Hubik have identified that inactive satellites create a feedback loop for collisions (2018). This element was also supported in the literature review, noting that the inability to accurately control inactive satellites result with a greater chance of collisions than active satellites. Active satellites, on the other hand, create a balancing loop, or the desired state. Active satellites reduce the likelihood of collisions, given their known trajectories and lifespans. The cascading collisions that occur between small, medium, and large however, create feedback loops, given the uncertain trajectories of these elements. Feedback loops are closed, suggesting that the collisions between these elements will continue unless a new element is introduced into the system. Using this information as a foundation, and considering the introduction of satellite maintenance, a new causal loop diagram was constructed to illustrate the potential impact this difference would have on collisions, as well as orbital decay. Research from the literature review also shed light on the importance of post mission procedures, which increases orbital decay and in turn, reduces orbital debris as well 
as the likelihood for future collisions. It was therefore included in the diagram. The new causal loop diagram is shown in Figure 3.

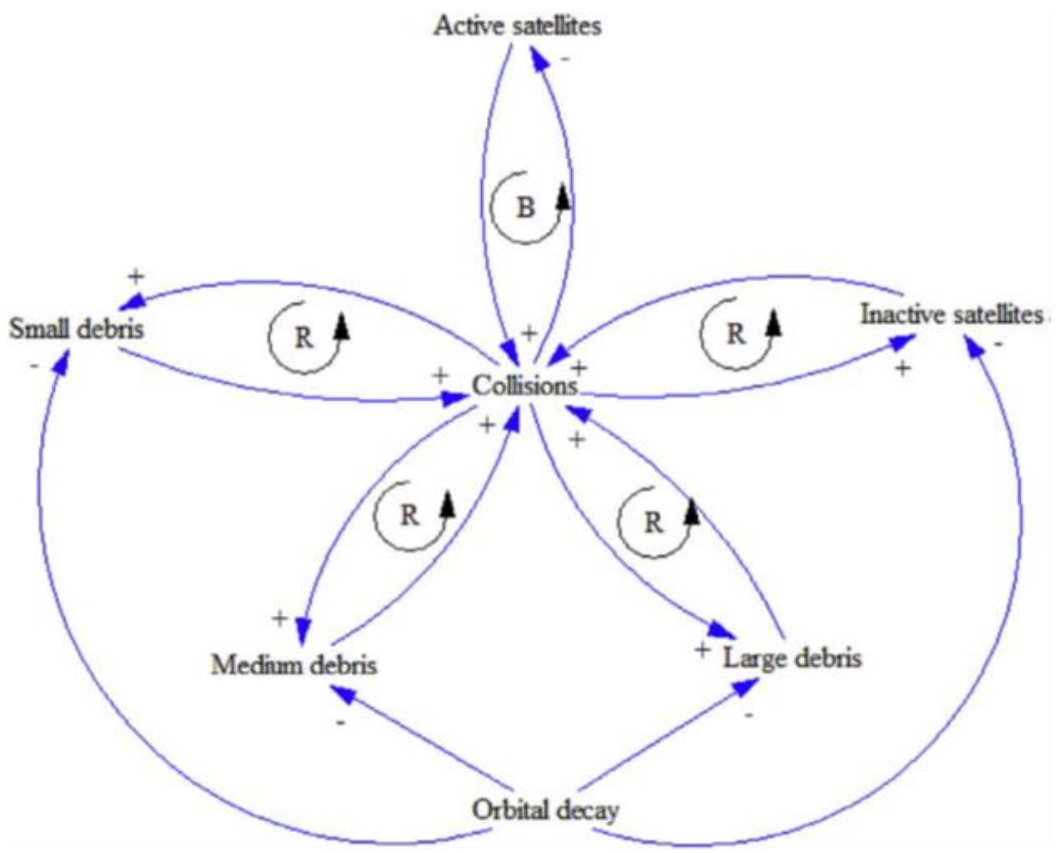

Figure 2. Casual loop diagram. Adapted from "Kessler Syndrome: System Dynamics Model," by J. Drmola, and T. Hubik, 2018, Space Policy, 44/45, p. 35. doi:10.1016/j.spacepol.2018.03.003 


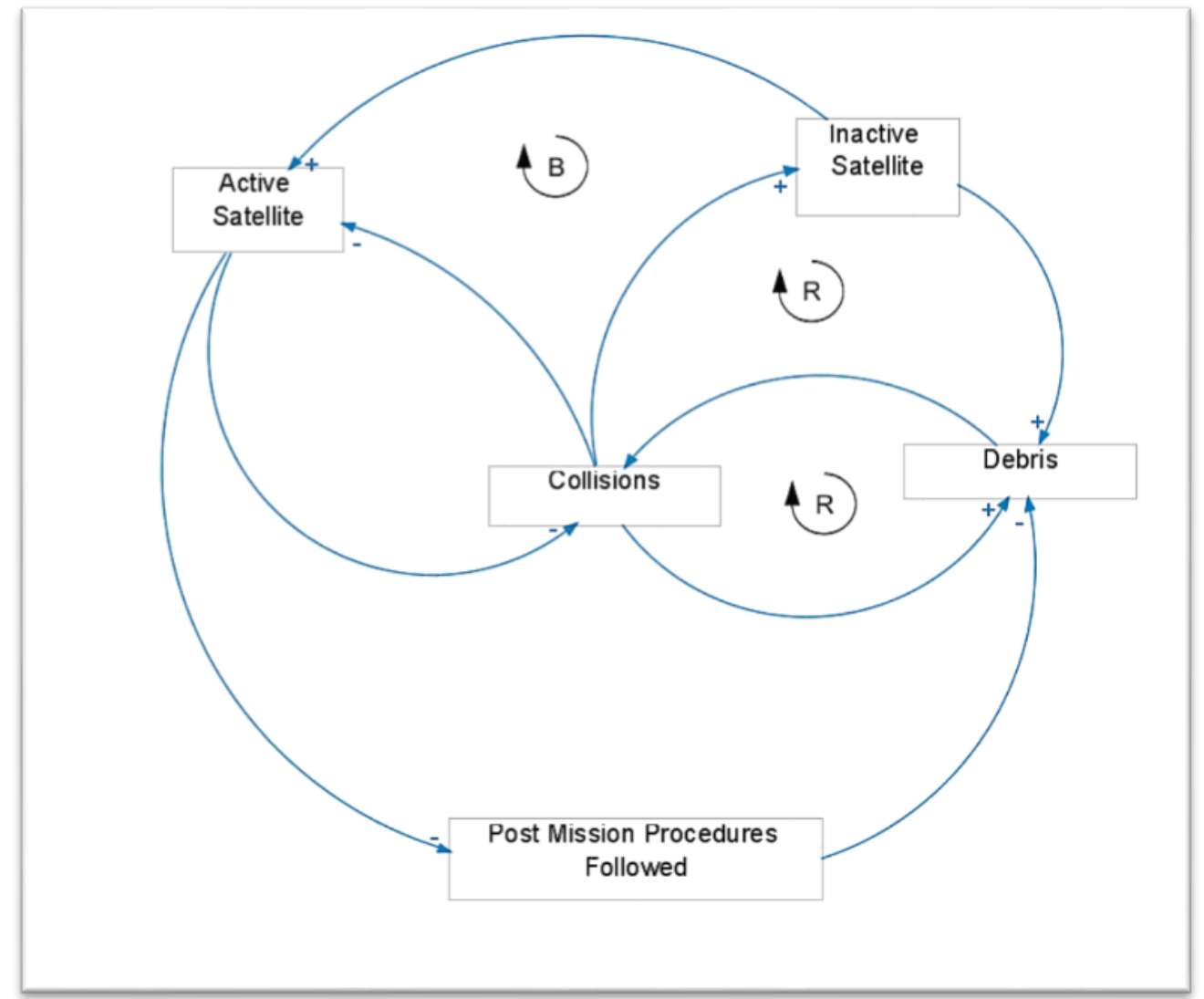

Figure 3. Causal loop diagram, accounting for the new relationship between inactive and active satellites. Image designed using Simantics software.

Figure 3 exhibits similar feedback loops between inactive satellites, collisions, and debris, but also includes a shift of inactive satellites to those that are active. In doing so, active satellites are increased, potential collisions are reduced, debris is reduced, and adherence to post mission procedures is increased.

In addition to causal loop diagrams, flowcharts were also used to illustrate the relationships between these elements. In Figure 1, Drmola and Hubik (2018) highlighted that when satellites become inoperable, it follows a path of disintegration, and subsequent fragmentation. The inclusion of post mission procedures, as well as the shift from inactive to active satellites, is shown in the new flowchart below (Figure 4). It is evident that increasing the number of active satellites, from the current pool of inactive satellites, increases the possibility of adherence to post mission procedures and long-term debris reduction. 


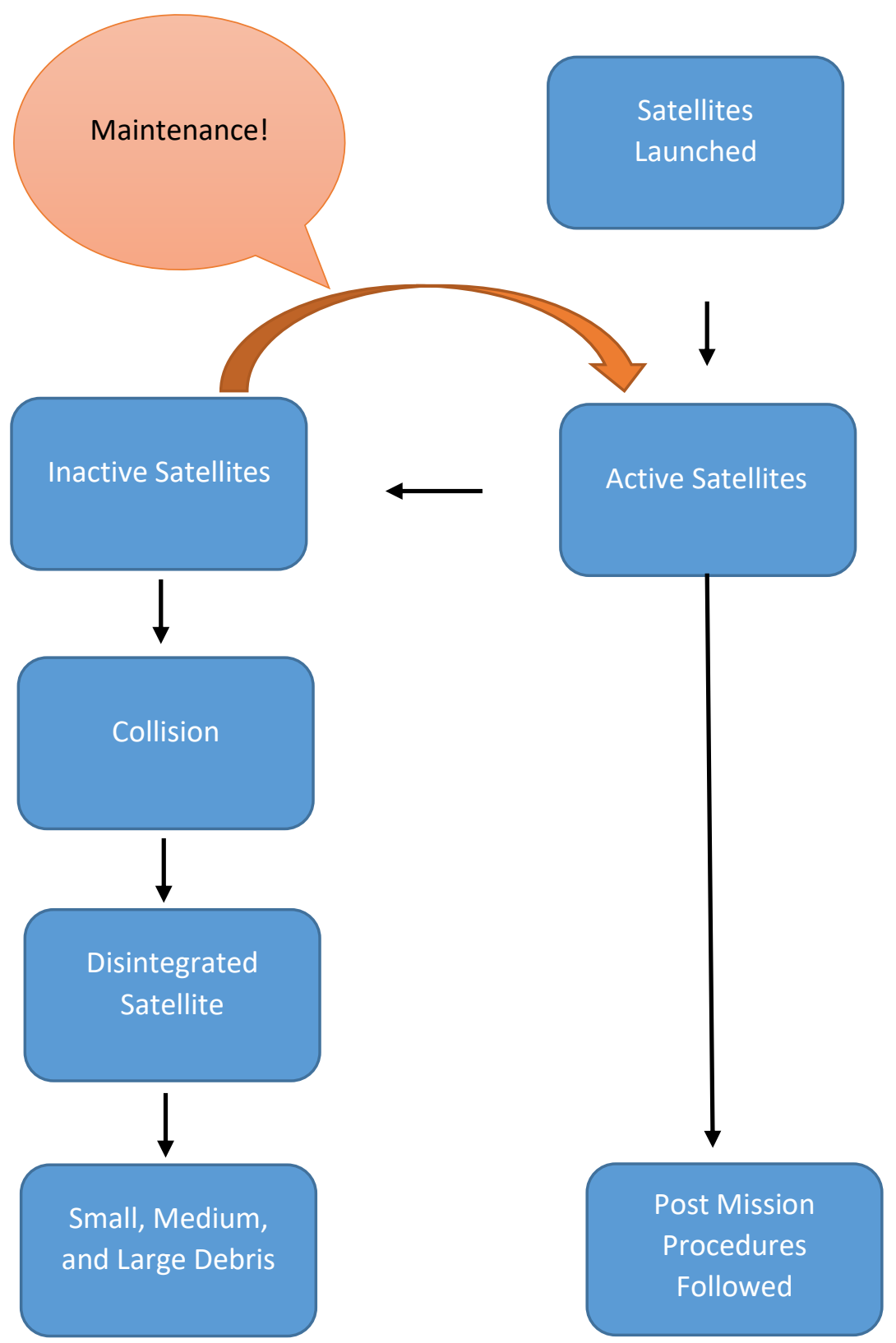

Figure 4. Updated flowchart illustrating new shift of inactive satellites to the active satellite group through inclusion of in-orbit maintenance.

\section{Conclusion and Recommendation}

After examining the data provided in the research analysis, it is clear that satellite maintenance can minimize the Kessler Effect, even if only indirectly. By shifting the number of inactive satellites to those that are active, it would create an 
environment which is less susceptible to collisions. While existing debris would continue to pose challenges, reducing the overall number of inactive satellites minimizes the chance of these satellites unintentionally colliding with other objects in orbit (active or inactive satellites, as well as debris).

Considering the potential for satellite maintenance to minimize the Kessler Effect, several recommendations are presented. The first is to strongly consider satellite maintenance as a viable opportunity to reduce space debris. There are many satellites which have simply run out of fuel or need to be repositioned. There are also many which demonstrate a significantly higher potential for collisions, due to their size and location. These elements must be weighed against each other in order to identify the satellites that are best suited for in-orbit maintenance (those that exhibit optimal maintenance faults and have the greatest likelihood for a collision). Satellite manufacturers must then begin developing smaller maintenance unmanned systems that are capable of performing such tasks. Given the extensive knowledge of the satellites themselves, it is appropriate to assume that they may also possess the potential to maintain them in space. Companies that could successfully do this may extend the lives of their satellites, which would justify the cost of the mission with additional revenue opportunities. Joint ventures may also prove to be a lucrative market for those that can capitalize on the current resources currently in space, but do not currently possess the technology to do so.

In addition to the manufacture of unmanned satellite maintenance systems, other supportive elements are needed. Firstly, an international agency similar to ICAO for the aviation industry, must support debris mitigation efforts. While it is clear that there is no single answer to the problem of orbital debris, and ultimately the impact of the Kessler Effect, there are many actions that could have a significant impact when viewed as a single, combined effort. The range in debris size suggests that many different approaches are needed. If each group had its own protocol for removal, and there was a supporting structure in place to do so, the Kessler Effect could truly be minimized, it not eliminated altogether.

Overall, orbital debris and the resulting impact on the Kessler Effect will continue to threaten the use of the orbits surrounding Earth. In order to ensure the long-term use of this space, mitigation efforts are needed. Requiring maintenance to be performed by those who are responsible for the satellite would not only extend the lives of these systems and bring in additional revenue, but also minimize the occurrence of collisions and orbital debris. 


\section{References}

Adilov, N., Alexander, P. J., \& Cunningham, B. M. (2018). An economic "Kessler syndrome:" A dynamic model of earth orbit debris. Economics Letters, 166, 79-82. doi:10.1016/j.econlet.2018.02.025

Anselmo, L., \& Pardini, C. (2017). Dimensional and scale analysis applied to the preliminary assessment of the environment criticality of large constellations in LEO. Acta Astronautica. doi:10.1016/j.actaastro.2017.09.028

Bonnal, C., Ruault, J., \& Desjean, M. (2013). Active debris removal: Recent progress and current trends. Acta Astronautica, 85, 51-60. doi:10.1016/j.actaastro.2012.11.009

Chu, X., Zhang, Y., Zhang, J., Lu, S., \& Sun, Y. (2016). Optimised collision avoidance for an ultra-close rendezvous with a failed satellite based on the gauss pseudospectral method. Acta Astronautica, 128, 363-376. doi:10.1016/j.actaastro.2016.07.011

Cour-Palais, B. G., \& Kessler, D. J. (1978). Collision frequency of artificial satellites - the creation of a debris belt. Journal of Geophysical Research, 83, 2637-2646. Retrieved from https://agupubs.onlinelibrary.wiley.com/ journal/21562202

Defense Advanced Research Projects Agency. (2018). Robotic servicing of geosynchronous satellites. Retrieved from https://www.darpa.mil /program/robotic-servicing-of-geosynchronous-satellites

Drmola, J., \& Hubik, T. (2018). Kessler syndrome: System dynamics model. Space Policy, 44/45, 29-39. doi:10.1016/j.spacepol.2018.03.003

European Space Agency. (2018a). Active debris removal. Retrieved from: https://m.esa.int/Our_Activities/Operations/Space_Debris/Active_debris_ removal

European Space Agency. (2018b). Space debris by the numbers. Retrieved from: https://www.esa.int/Our_Activities/Operations/Space_Debris/Space_ debris_by _the_numbers

Forshaw, J. L., Aglietti, G. S., Navarathinam, N., Kadhem, H., Salmon, T., Pisseloup, A., . . . Steyn, W. H. (2016). RemoveDEBRIS: An in-orbit active debris removal demonstration mission. Acta Astronautica, 127, 448-463. doi:10.1016/j.actaastro.2016.06.018

Gonzalo, J., Domínguez, D., \& López, D. (2014). On the challenge of a century lifespan satellite. Progress in Aerospace Sciences, 70, 28-41. doi:10.1016/j.paerosci.2014.05.001

Hull, S. (2013). NASA technical reports server, Chapter 30, End of mission considerations. Retrieved from https://ntrs.nasa.gov/archive/nasa/ casi.ntrs.nasa.gov/20130000278.pdf 
Inter-Agency Space Debris Coordination Committee. (2016). Member agencies. Retrieved from: https://www.iadc-online.org/

Kelly, F. P., \& Holm, T. M. (2014). Landsat: Sustaining earth observations beyond Landsat 8. Photogrammetric Engineering and Remote Sensing, 80(1), 15. Retrieved from https://pubs.er.usgs.gov/publication/70187766

Khokhlachev, E. N. (2011). Cost optimization for maintaining the survivability of terrestrial objects of a satellite control system. Solar System Research, 45(7), 641-644. doi:10.1134/S0038094611070112

Liou, J. (2011). An active debris removal parametric study for LEO environment remediation. Advances in Space Research, 47(11), 1865-1876. doi:10.1016/j.asr.2011.02.003

National Aeronautics and Space Administration. (2018a). NASA's ODPO's large constellation study. Orbital debris quarterly news. Retrieved from https://www.orbitaldebris.jsc.nasa.gov/quarterly-news/pdfs/odqnv22i3.pdf

National Aeronautics and Space Administration. (2018b). Orbital debris program office. Retrieved from: https://www.orbitaldebris.jsc.nasa.gov/mitigation/

National Aeronautics and Space Administration. (2018c). Restore-L robotic servicing mission. Retrieved from: https://sspd.gsfc.nasa.gov/restore1.html

OneWeb (2019). Responsible use of space. OneWeb World, online. Retrieved from: http://www.oneweb.world/

Qi, R., Misra, A. K., \& Zuo, Z. (2017). Active debris removal using doubletethered space-tug system. Journal of Guidance, Control, and Dynamics, 40(3), 722-730. doi:10.2514/1.G000699

Russell, K. (2017). Satellite launches to increase threefold over the next decade. Via Satellite, innovation. Retrieved from https://www.satellitetoday.com/ innovation/ 2017/10/12/satellite-launches-increase-threefold-next-decade/

Simantics (2019). Why Simantics? Retrieved from https:/www.simantics.org/

Tummala, A. R., \& Dutta, A. (2017). An overview of cube-satellite propulsion technologies and trends. Aerospace, 4(4), 58. doi:10.3390/aerospace4040058

Valverde, A., \& Tsiotras, P. (2018). Spacecraft robot kinematics using dual quaternions. Robotics, 7(4), 64. doi:10.3390/robotics7040064

Vavrin, A. B., Matney, M. J., \& Manis, A. P. (2017). Effects of CubeSat deployments in low-Earth orbit. Retrieved from https://conference.sdo.esoc.esa.int/proceedings/ sdc7/paper/509

Werner, D. (2018). ESA to investigate links between debris removal and satellite servicing. Spacenews. Retrieved from https://spacenews.com/esa-toinvestigate-links-between-debris-removal-and-satellite-servicing/ 